PROCEEDINGS OF THE

AMERICAN MATHEMATICAL SOCIETY

Volume 135, Number 6, June 2007, Pages 1817-1827

S 0002-9939(06)08668-0

Article electronically published on November 7, 2006

\title{
INVERSE SCATTERING TRANSFORM FOR THE TODA HIERARCHY WITH QUASI-PERIODIC BACKGROUND
}

\author{
IRYNA EGOROVA, JOHANNA MICHOR, AND GERALD TESCHL
}

(Communicated by Joseph A. Ball)

\begin{abstract}
We provide a rigorous treatment of the inverse scattering transform for the entire Toda hierarchy in the case of a quasi-periodic finite-gap background solution.
\end{abstract}

\section{INTRODUCTION}

Since the seminal work of Gardner et al. 7] in 1967 the inverse scattering transform is one of the main tools for solving completely integrable wave equations, and numerous articles have been devoted to this subject since then. However, with only a few exceptions, only the case of short range perturbations of a constant background solution has been investigated. The first to consider a non-constant background seem to be Kuznetsov and A.V. Mikhallov, [8], who informally treated the Korteweg-de Vries equation with the Weierstraß elliptic function as stationary background solution. Formulas for arbitrary periodic background were given by Firsova in [5]. However, the discontinuous behavior at the band edges is not investigated there and neither is the question of when the time dependent scattering data satisfy the hypothesis necessary for the Gel'fand-Levitan-Marchenko theory (see, e.g., 10 for the constant background case). Moreover, the normalization chosen there blows up when the eigenvalues lie at certain positions in the gaps.

Our aim in the present paper is to provide a rigorous treatment of the inverse scattering transform for the Toda hierarchy in the case of quasi-periodic finitegap background solutions (including the case of arbitrary periodic solutions). We include a discussion of the problems arising from the poles of the Baker-Akhiezer functions at the Dirichlet eigenvalues (which are absent in the constant background case). In the constant background case this was first done for the Toda equation by Flaschka in [6] on an informal level. The first rigorous treatment for the entire Toda hierarchy was given by one of us in [12] (see also [4, 15], 14]). For further results on solutions of the Toda equation with non-trivial spatial asymptotics see [1].

After introducing the Toda hierarchy in Section 2, we will first show that a solution will stay close to a given background solution in Section 3 , This result

Received by the editors December 1, 2005 and, in revised form, February 7, 2006.

2000 Mathematics Subject Classification. Primary 37K15, 37K10; Secondary 47B36, 34L25.

Key words and phrases. Inverse scattering, Toda hierarchy, periodic.

This work was supported by the Austrian Science Fund (FWF) under Grant No. P17762 and INTAS Research Network NeCCA 03-51-6637. 
implies that a short-range perturbation of a quasi-periodic finite-gap solution will stay short range for all time, and it shows that the time-dependent scattering data satisfy the hypothesis necessary for the Gel'fand-Levitan-Marchenko theory. This result constitutes the main technical ingredient for the inverse scattering transform. In Section 4 we review some necessary facts on quasi-periodic finite-gap solutions and in Section 5 we compute the time dependence of the scattering data and discuss its dynamics. Finally we establish the conserved quantities of the Toda hierarchy in this context.

\section{THE TODA HIERARCHY}

In this section we introduce the Toda hierarchy using the standard Lax formalism ([9]). We first review some basic facts from [2] (see also [14]).

We will only consider bounded solutions and hence require

Hypothesis $\mathbf{H}$.2.1. Suppose $a(t), b(t)$ satisfy

$$
a(t) \in \ell^{\infty}(\mathbb{Z}, \mathbb{R}), \quad b(t) \in \ell^{\infty}(\mathbb{Z}, \mathbb{R}), \quad a(n, t) \neq 0, \quad(n, t) \in \mathbb{Z} \times \mathbb{R},
$$

and let $t \mapsto(a(t), b(t))$ be differentiable in $\ell^{\infty}(\mathbb{Z}) \oplus \ell^{\infty}(\mathbb{Z})$.

Associated with $a(t), b(t)$ is a Jacobi operator

$$
H(t): \ell^{2}(\mathbb{Z}) \rightarrow \ell^{2}(\mathbb{Z}), \quad f \mapsto \tau(t) f,
$$

where

$$
\tau(t) f(n)=a(n, t) f(n+1)+a(n-1, t) f(n-1)+b(n, t) f(n)
$$

and $\ell^{2}(\mathbb{Z})$ denotes the Hilbert space of square summable (complex-valued) sequences over $\mathbb{Z}$. Moreover, choose constants $c_{0}=1, c_{j}, 1 \leq j \leq r, c_{r+1}=0$, set

$$
\begin{aligned}
& g_{j}(n, t)=\sum_{\ell=0}^{j} c_{j-\ell}\left\langle\delta_{n}, H(t)^{\ell} \delta_{n}\right\rangle, \\
& h_{j}(n, t)=2 a(n, t) \sum_{\ell=0}^{j} c_{j-\ell}\left\langle\delta_{n+1}, H(t)^{\ell} \delta_{n}\right\rangle+c_{j+1},
\end{aligned}
$$

and consider the Lax operator

$$
P_{2 r+2}(t)=-H(t)^{r+1}+\sum_{j=0}^{r}\left(2 a(t) g_{j}(t) S^{+}-h_{j}(t)\right) H(t)^{r-j}+g_{r+1}(t),
$$

where $S^{ \pm} f(n)=f(n \pm 1)$. Restricting to the two-dimensional nullspace

$$
\operatorname{Ker}(\tau(t)-z), z \in \mathbb{C},
$$

of $\tau(t)-z$, we have the following representation of $P_{2 r+2}(t)$ :

$$
\left.P_{2 r+2}(t)\right|_{\operatorname{Ker}(\tau(t)-z)}=2 a(t) G_{r}(z, t) S^{+}-H_{r+1}(z, t),
$$

where $G_{r}(z, n, t)$ and $H_{r+1}(z, n, t)$ are monic polynomials in $z$ of the type

$$
\begin{aligned}
G_{r}(z, n, t) & =\sum_{j=0}^{r} z^{j} g_{r-j}(n, t), \\
H_{r+1}(z, n, t) & =z^{r+1}+\sum_{j=0}^{r} z^{j} h_{r-j}(n, t)-g_{r+1}(n, t) .
\end{aligned}
$$


A straightforward computation shows that the Lax equation

$$
\frac{d}{d t} H(t)-\left[P_{2 r+2}(t), H(t)\right]=0, \quad t \in \mathbb{R},
$$

is equivalent to

$$
\begin{aligned}
& \mathrm{TL}_{r}(a(t), b(t))_{1}=\dot{a}(t)-a(t)\left(g_{r+1}^{+}(t)-g_{r+1}(t)\right)=0, \\
& \mathrm{TL}_{r}(a(t), b(t))_{2}=\dot{b}(t)-\left(h_{r+1}(t)-h_{r+1}^{-}(t)\right)=0,
\end{aligned}
$$

where the dot denotes a derivative with respect to $t$ and $f^{ \pm}(n)=f(n \pm 1)$. Varying $r \in \mathbb{N}_{0}$ yields the Toda hierarchy $\operatorname{TL}_{r}(a, b)=\left(\mathrm{TL}_{r}(a, b)_{1}, \mathrm{TL}_{r}(a, b)_{2}\right)=0$. We will always consider $r$ as a fixed, but arbitrary, value.

Finally, we recall that the Lax equation (2.7) implies existence of a unitary propagator $U_{r}(t, s)$ such that the family of operators $H(t), t \in \mathbb{R}$, are unitarily equivalent, $H(t)=U_{r}(t, s) H(s) U_{r}(s, t)$.

\section{The initial Value PRoblem}

First of all we recall the basic existence and uniqueness theorem for the Toda hierarchy (see, e.g., [12, [13, or [14, Section 12.2]).

Theorem 3.1. Suppose $\left(a_{0}, b_{0}\right) \in M=\ell^{\infty}(\mathbb{Z}) \oplus \ell^{\infty}(\mathbb{Z})$. Then there exists a unique integral curve $t \mapsto(a(t), b(t))$ in $C^{\infty}(\mathbb{R}, M)$ of the Toda hierarchy, that is, $\mathrm{TL}_{r}(a(t), b(t))=0$, such that $(a(0), b(0))=\left(a_{0}, b_{0}\right)$.

In 12] it was shown that solutions which are asymptotically close to the constant solution at the initial time stay close for all time. Our first aim is to extend this result to include perturbations of quasi-periodic finite-gap solutions. In fact, we will even be a bit more general.

Lemma 3.2. Suppose $a(n, t), b(n, t)$ and $\bar{a}(n, t), \bar{b}(n, t)$ are two arbitrary bounded solutions of the Toda hierarchy satisfying (3.1) for one $t_{0} \in \mathbb{R}$. Then (3.1) holds for all $t \in \mathbb{R}$, that is,

$$
\sum_{n \in \mathbb{Z}} w(n)(|a(n, t)-\bar{a}(n, t)|+|b(n, t)-\bar{b}(n, t)|)<\infty,
$$

where $w(n)>0$.

Proof. Without loss of generality we assume that $t_{0}=0$. Consider the expression

$$
\|(a(t), b(t))\|_{*}=\sum_{n \in \mathbb{Z}} w(n)(|a(n, t)-\bar{a}(n, t)|+|b(n, t)-\bar{b}(n, t)|)
$$

which remains finite at least for small $t$ since there is a local solution of the Toda hierarchy with respect to $\|\cdot\|_{*}$. We claim that the following estimate holds:

$$
\begin{aligned}
& \sum_{n \in \mathbb{Z}} w(n)\left|g_{r}(n, t)-\bar{g}_{r}(n, t)\right| \leq C_{r}\|(a(t), b(t))\|_{*}, \\
& \sum_{n \in \mathbb{Z}} w(n)\left|h_{r}(n, t)-\bar{h}_{r}(n, t)\right| \leq C_{r}\|(a(t), b(t))\|_{*},
\end{aligned}
$$

where $C_{r}=C(\|H(0)\|,\|\bar{H}(0)\|)$ is a positive constant due to Hypothesis $\mathrm{H} 2.1$, Let us prove (3.2) by induction on $r$. It suffices to consider the case where $c_{j}=0$, $1 \leq j \leq r$, since all involved sums are finite. In this case [14, Lemma 6.4] shows 
that $g_{j}(n, t), h_{j}(n, t)$ can be recursively computed from $g_{0}(n, t)=1, h_{0}(n, t)=0$ via

$$
\begin{aligned}
& g_{j+1}(n, t)=\frac{1}{2}\left(h_{j}(n, t)+h_{j}(n-1, t)\right)+b(n, t) g_{j}(n, t), \\
& h_{j+1}(n, t)=2 a(n, t)^{2} \sum_{l=0}^{j} g_{j-l}(n, t) g_{l}(n+1, t)-\frac{1}{2} \sum_{l=0}^{j} h_{j-l}(n, t) h_{l}(n, t) .
\end{aligned}
$$

Hence

$$
g_{1}(n, t)-\bar{g}_{1}(n, t)=b(n, t)-\bar{b}(n, t), \quad h_{1}(n, t)-\bar{h}_{1}(n, t)=2\left(a(n, t)^{2}-\bar{a}(n, t)^{2}\right)
$$

and

$$
\begin{aligned}
& g_{r+1}(n, t)-\bar{g}_{r+1}(n, t)=(b(n, t)-\bar{b}(n, t)) g_{r}(n, t)+\bar{b}(n, t)\left(g_{r}(n, t)-\bar{g}_{r}(n, t)\right) \\
& \quad+\frac{1}{2}\left(h_{r}(n, t)-\bar{h}_{r}(n, t)+h_{r}(n-1, t)-\bar{h}_{r}(n-1, t)\right), \\
& h_{r+1}(n, t)-\bar{h}_{r+1}(n, t)=\frac{1}{2} \sum_{l=0}^{r}\left(h_{r-l}(n, t) h_{l}(n, t)-\bar{h}_{r-l}(n, t) \bar{h}_{l}(n, t)\right) \\
& \quad+2 a(n, t)^{2} \sum_{l=0}^{r} g_{r-l}(n, t) g_{l}(n+1, t)-2 \bar{a}(n, t)^{2} \sum_{l=0}^{r} \bar{g}_{r-l}(n, t) \bar{g}_{l}(n+1, t) .
\end{aligned}
$$

Since both sequences $a, b$ and $\bar{a}, \bar{b}$ are solutions of the Toda hierarchy, (2.8) yields

$$
\begin{aligned}
& \dot{a}(t)-\dot{\bar{a}}(t)=a(t)\left(g_{r+1}^{+}(t)-g_{r+1}(t)\right)-\bar{a}(t)\left(\bar{g}_{r+1}^{+}(t)-g_{r+1}(t)\right) \\
& \quad=(a(t)-\bar{a}(t))\left(\bar{g}_{r+1}^{+}(t)-\bar{g}_{r+1}(t)\right)-a(t)\left(\bar{g}_{r+1}^{+}(t)-g_{r+1}^{+}(t)+g_{r+1}(t)-\bar{g}_{r+1}(t)\right)
\end{aligned}
$$

and thus

$$
\begin{aligned}
& |a(n, t)-\bar{a}(n, t)| \leq|a(n, 0)-\bar{a}(n, 0)|+C_{1} \int_{0}^{t}|a(n, s)-\bar{a}(n, s)| d s \\
& \quad+\|H(0)\| \int_{0}^{t}\left(\left|g_{r+1}(n+1, s)-\bar{g}_{r+1}(n+1, s)\right|+\left|g_{r+1}(n, s)-\bar{g}_{r+1}(n, s)\right|\right) d s \\
& \quad|b(n, t)-\bar{b}(n, t)| \leq|b(n, 0)-\bar{b}(n, 0)| \\
& \quad+\int_{0}^{t}\left(\left|h_{r+1}(n, s)-\bar{h}_{r+1}(n, s)\right|+\left|h_{r+1}(n-1, s)-\bar{h}_{r+1}(n-1, s)\right|\right) d s .
\end{aligned}
$$

By (3.2), $\|(a(t), b(t))\|_{*} \leq\|(a(0), b(0))\|_{*}+C \int_{0}^{t}\|(a(s), b(s))\|_{*} d s$, where $C=$ $\left(C_{1}+2\|H(0)\|+2\right) C_{r+1}$. Finally, applying Gronwall's inequality

$$
\|(a(t), b(t))\|_{*} \leq\|(a(0), b(0))\|_{*} \exp (C t)
$$

finishes the proof.

\section{QUASI-PERIODIC FINITE-GAP SOLUTIONS}

As a preparation for our next section we first need to recall some facts on quasiperiodic finite-gap solutions (again see 2] or [14]).

Let $\mathbb{M}$ be the Riemann surface associated with the following function:

$$
R_{2 g+2}^{1 / 2}(z)=-\prod_{j=0}^{2 g+1} \sqrt{z-E_{j}}, \quad E_{0}<E_{1}<\cdots<E_{2 g+1},
$$


where $g \in \mathbb{N}$ and $\sqrt{ }$. is the standard root with branch cut along $(-\infty, 0)$. $\mathbb{M}$ is a compact, hyperelliptic Riemann surface of genus $g$. A point on $\mathbb{M}$ is denoted by $p=\left(z, \pm R_{2 g+2}^{1 / 2}(z)\right)=(z, \pm), z \in \mathbb{C}$, or $p=\infty_{ \pm}$, and the projection onto $\mathbb{C} \cup\{\infty\}$ by $\pi(p)=z$.

Now pick $g$ numbers (the Dirichlet eigenvalues)

$$
\left(\hat{\mu}_{j}\right)_{j=1}^{g}=\left(\mu_{j}, \sigma_{j}\right)_{j=1}^{g}
$$

whose projections lie in the spectral gaps, that is, $\mu_{j} \in\left[E_{2 j-1}, E_{2 j}\right]$. Associated with these numbers is the divisor $\mathcal{D}_{\hat{\mu}}$ which is one at the points $\hat{\mu}_{j}$ and zero else. Using this divisor we introduce

$$
\begin{aligned}
\underline{z}(p, n, t) & =\underline{\hat{A}}_{p_{0}}(p)-\underline{\hat{\alpha}}_{p_{0}}\left(\mathcal{D}_{\hat{\hat{\mu}}}\right)-n \underline{\hat{A}}_{\infty_{-}}\left(\infty_{+}\right)+t \underline{U}_{s}-\hat{\underline{\Xi}}_{p_{0}} \in \mathbb{C}^{g}, \\
\underline{z}(n, t) & =\underline{z}\left(\infty_{+}, n, t\right),
\end{aligned}
$$

where $\Xi_{p_{0}}$ is the vector of Riemann constants, $\underline{U}_{s}$ the $b$-periods of the Abelian differential $\Omega_{s}$ defined below, and $\underline{A}_{p_{0}}\left(\underline{\alpha}_{p_{0}}\right)$ is Abel's map (for divisors). The hat indicates that we regard it as a (single-valued) map from $\hat{\mathbb{M}}$ (the fundamental polygon associated with $\mathbb{M})$ to $\mathbb{C}^{g}$. We recall that the function $\theta(\underline{z}(p, n, t))$ has precisely $g$ zeros $\hat{\mu}_{j}(n, t)$ (with $\hat{\mu}_{j}(0,0)=\hat{\mu}_{j}$ ), where $\theta(\underline{z})$ is the Riemann theta function of $\mathbb{M}$.

Taking a stationary solution of $\mathrm{TL}_{g}$ with constants $c_{j}, 1 \leq j \leq g$, as the initial condition for another equation $\widehat{\mathrm{TL}}_{s}$ with constants $\hat{c}_{j}, 1 \leq j \leq s$, in the Toda hierarchy (2.8) one obtains the quasi-periodic finite gap solutions of the Toda hierarchy given by (see [14, Sections 13.1, 13.2])

$$
\begin{aligned}
a_{q}(n, t)^{2} & =\tilde{a}^{2} \frac{\theta(\underline{z}(n+1, t)) \theta(\underline{z}(n-1, t))}{\theta(\underline{z}(n, t))^{2}}, \\
b_{q}(n, t) & =\tilde{b}+\left.\sum_{j=1}^{g} c_{j}(g) \frac{\partial}{\partial w_{j}} \ln \left(\frac{\theta(\underline{w}+\underline{z}(n, t))}{\theta(\underline{w}+\underline{z}(n-1, t))}\right)\right|_{\underline{w}=0} .
\end{aligned}
$$

The constants $\tilde{a}, \tilde{b}, c_{j}(g)$ depend only on the Riemann surface (see [14, Section 9.2]). Introduce

$$
\begin{aligned}
& \phi_{q}(p, n, t)=C(n, t) \frac{\theta(\underline{z}(p, n+1, t))}{\theta(\underline{z}(p, n, t))} \exp \left(\int_{p_{0}}^{p} \omega_{\infty_{+}, \infty_{-}}\right), \\
& \psi_{q}(p, n, t)=C(n, 0, t) \frac{\theta(\underline{z}(p, n, t))}{\theta(\underline{z}(p, 0,0))} \exp \left(n \int_{p_{0}}^{p} \omega_{\infty_{+}, \infty_{-}}+t \int_{p_{0}}^{p} \Omega_{s}\right),
\end{aligned}
$$

where $C(n, t), C(n, 0, t)$ are real-valued,

$$
C(n, t)^{2}=\frac{\theta(\underline{z}(n-1, t))}{\theta(\underline{z}(n+1, t))}, \quad C(n, 0, t)^{2}=\frac{\theta(\underline{z}(0,0)) \theta(\underline{z}(-1,0))}{\theta(\underline{z}(n, t)) \theta(\underline{z}(n-1, t))},
$$

and the sign of $C(n, t)$ is opposite to that of $a_{q}(n, t) . \omega_{\infty_{+}, \infty_{-}}$is the Abelian differential of the third kind with poles at $\infty_{+}$, respectively $\infty_{-}$, and $\Omega_{s}$ is an Abelian differential of the second kind with poles at $\infty_{+}$, respectively $\infty_{-}$, whose Laurent expansion is given by the coefficients $(j+1) \hat{c}_{s-j}$ associated with $\widehat{\mathrm{TL}}_{s}$ (see 
14. Sections 13.1, 13.2]). Then

$$
\begin{aligned}
\tau_{q}(t) \psi_{q}(p, n, t) & =\pi(p) \psi_{q}(p, n, t), \\
\frac{d}{d t} \psi_{q}(p, n, t) & =2 a_{q}(n, t) \hat{G}_{s}(p, n, t) \psi_{q}(p, n+1, t)-\hat{H}_{s+1}(p, n, t) \psi_{q}(p, n, t) \\
& =\hat{P}_{q, 2 s+2}(t) \psi_{q}(p, n, t)(n),
\end{aligned}
$$

where we use the hat to distinguish the quantities associated with $\widehat{\mathrm{TL}}_{s}$ from those associated with $\mathrm{TL}_{g}$.

It is well known that the spectrum of $H_{q}(t)$ is time independent and consists of $g+1$ bands

$$
\sigma\left(H_{q}\right)=\bigcup_{j=0}^{g}\left[E_{2 j}, E_{2 j+1}\right]
$$

For further information and proofs we refer to [14, Chapter 9].

Next let us renormalize the Baker-Akhiezer function

$$
\tilde{\psi}_{q}(p, n, t)=\frac{\psi_{q}(p, n, t)}{\psi_{q}(p, 0, t)}
$$

such that $\tilde{\psi}_{q}(p, 0, t)=1$ and let us define $\alpha_{s}(p, t)$ via

$$
\exp \left(\alpha_{s}(p, t)\right)=\psi_{q}(p, 0, t)=C(0,0, t) \frac{\theta(\underline{z}(p, 0, t))}{\theta(\underline{z}(p, 0,0))} \exp \left(t \int_{p_{0}}^{p} \Omega_{s}\right) .
$$

One can show $(14,(13.49))$

$$
\begin{aligned}
\exp \left(\alpha_{s}(p, t)\right) & =\exp \left(\int_{0}^{t}\left(2 a_{q}(0, x) \hat{G}_{s}(p, 0, x) \phi_{q}(p, 0, x)-\hat{H}_{s+1}(p, 0, x)\right) d x\right) \\
& =\sqrt{\frac{G_{g}(p, 0, t)}{G_{g}(p, 0,0)}} \exp \left(R_{2 g+2}^{1 / 2}(p) \int_{0}^{t} \frac{\hat{G}_{s}(p, 0, x)}{G_{g}(p, 0, x)} d x\right),
\end{aligned}
$$

where

$$
G_{g}(z, n, t)=\prod_{j=1}^{g}\left(z-\mu_{j}(n, t)\right)
$$

However, let us emphasize that the integrals in (4.10) are only well defined until we hit the first pole of the integrand. After that time they have to be interpreted as principal values (compare also Remark 5.4(i)), respectively, (4.9) has to be used.

In addition, observe

$$
\begin{aligned}
& \exp \left(\alpha_{s,+}(z, t)+\alpha_{s,-}(z, t)\right)=\frac{G_{g}(z, 0, t)}{G_{g}(z, 0,0)}, \\
& \exp \left(\alpha_{s,+}(z, t)-\alpha_{s,-}(z, t)\right)=\exp \left(2 R_{2 g+2}^{1 / 2}(z) \int_{0}^{t} \frac{\hat{G}_{s}(z, 0, x)}{G_{g}(z, 0, x)} d x\right),
\end{aligned}
$$

where $\alpha_{s, \pm}$ are the two branches of $\alpha_{s}$. For $\lambda \in \sigma\left(H_{q}\right)$ we have

$$
\overline{\alpha_{s, \pm}(\lambda, t)}=\alpha_{s, \mp}(\lambda, t),
$$

but note that $\alpha_{s, \mp}(\lambda, t) \neq \mp \alpha_{s, \pm}(\lambda, t)$ unless $g=0$. 


\section{INVERSE SCATTERING TRANSFORM}

Let $a(n, t), b(n, t)$ be a solution of the Toda hierarchy satisfying

$$
\sum_{n \in \mathbb{Z}}|n|\left(\left|a(n, t)-a_{q}(n, t)\right|+\left|b(n, t)-b_{q}(n, t)\right|\right)<\infty
$$

for one (and hence for any) $t_{0} \in \mathbb{R}$. In 3 . (see also [16]) we develop scattering theory for the Jacobi operator $H$ associated with $a(n), b(n)$. Jost solutions, transmission and reflection coefficients now depend on an additional parameter $t \in \mathbb{R}$. More precisely,

$$
\sigma(H(t)) \equiv \sigma(H), \quad \sigma_{e s s}(H)=\sigma\left(H_{q}\right), \quad \sigma_{p}(H)=\left\{\rho_{j}\right\}_{j=1}^{q} \subseteq \mathbb{R} \backslash \sigma\left(H_{q}\right),
$$

where $q \in \mathbb{N}$ is finite. The Jost solutions $\psi_{ \pm}(z, n, t)$ are normalized such that

$$
\tilde{\psi}_{ \pm}(z, n, t)=\tilde{\psi}_{q, \pm}(z, n, t)(1+o(1)) \quad \text { as } n \rightarrow \pm \infty .
$$

Transmission $T(\lambda, t)$ and reflection $R_{ \pm}(\lambda, t)$ coefficients are defined via the scattering relations

$$
T(\lambda, t) \tilde{\psi}_{ \pm}(\lambda, n, t)=\overline{\tilde{\psi}_{\mp}(\lambda, n, t)}+R_{\mp}(\lambda, t) \tilde{\psi}_{\mp}(\lambda, n, t), \quad \lambda \in \sigma\left(H_{q}\right),
$$

and the norming constants $\gamma_{ \pm, j}(t)$ corresponding to $\rho_{j} \in \sigma_{p}(H)$ are given by

$$
\gamma_{ \pm, j}(t)^{-1}=\sum_{n \in \mathbb{Z}}\left|\tilde{\psi}_{ \pm}\left(\rho_{j}, n, t\right)\right|^{2}
$$

To avoid the poles of the Baker-Akhiezer function, we will assume that none of the eigenvalues $\rho_{j}$ coincides with a Dirichlet eigenvalue $\mu_{k}(0,0)$. This can be done without loss of generality by shifting the initial time $t_{0}=0$ if necessary.

Remark 5.1. Due to this assumption there is no need to remove these poles for the definition of $\gamma_{ \pm, j}$, as we did in 3. Since the Dirichlet eigenvalues rotate in their gap, the factor needed to remove the poles would only unnecessarily complicate the time evolution of the norming constants. Moreover, these factors would eventually cancel in the Gel'fand-Levitan-Marchenko equation, which is the only interesting object from the inverse spectral point of view in the first place. See Remark 5.4 below.

Lemma 5.2. Let $(a(t), b(t))$ be a solution of the Toda hierarchy such that (5.1) holds. The functions

$$
\psi_{ \pm}(z, n, t)=\exp \left(\alpha_{s, \pm}(z, t)\right) \tilde{\psi}_{ \pm}(z, n, t)
$$

satisfy

$$
H(t) \psi_{ \pm}(z, n, t)=z \psi_{ \pm}(z, n, t), \quad \frac{d}{d t} \psi_{ \pm}(z, n, t)=\hat{P}_{2 s+2}(t) \psi_{ \pm}(z, n, t)
$$

Proof. We proceed as in [12, Theorem 3.2]. The Jost solutions $\psi_{ \pm}(z, n, t)$ are continuously differentiable with respect to $t$ by the same arguments as for $z$ (compare [3. Theorem 4.2]), and the derivatives are equal to the derivatives of the BakerAkhiezer functions as $n \rightarrow \pm \infty$.

For $z \in \rho(H)$, the solution $u_{ \pm}(z, n, t)$ of (5.6) with initial condition $\psi_{ \pm}(z, n, 0) \in$ $\ell_{ \pm}^{2}(\mathbb{Z})$ remains square summable near $\pm \infty$ for all $t \in \mathbb{R}$ (see [13] or [14, Lemma 12.16]), that is, $u_{ \pm}(z, n, t)=C_{ \pm}(t) \psi_{ \pm}(z, n, t)$. Letting $n \rightarrow \pm \infty$ we see $C_{ \pm}(t)=1$. The general result for all $z \in \mathbb{C}$ now follows from continuity. 
This implies

Theorem 5.3. Let $(a(t), b(t))$ be a solution of the Toda hierarchy such that (5.1) holds. The time evolution for the scattering data is given by

$$
\begin{aligned}
T(\lambda, t) & =T(\lambda, 0), \\
R_{ \pm}(\lambda, t) & =R_{ \pm}(\lambda, 0) \exp \left( \pm\left(\alpha_{s,+}(\lambda, t)-\alpha_{s,-}(\lambda, t)\right)\right), \\
\gamma_{ \pm, j}(t) & =\gamma_{ \pm, j}(0) \exp \left(2 \alpha_{s, \pm}\left(\rho_{j}, t\right)\right), \quad 1 \leq j \leq q .
\end{aligned}
$$

Proof. Since $\overline{\alpha_{s, \pm}(\lambda, t)}=\alpha_{s, \mp}(\lambda, t)$ by (4.13), we have

$$
\begin{aligned}
T(\lambda, t) & =\frac{W\left(\tilde{\psi}_{-}(\lambda, t), \overline{\tilde{\psi}_{-}(\lambda, t)}\right)}{W\left(\tilde{\psi}_{-}(\lambda, t), \tilde{\psi}_{+}(\lambda, t)\right)} \\
& =\frac{\exp \left(\alpha_{s,-}(\lambda, t)\right) \exp \left(\alpha_{s,+}(\lambda, t)\right)}{\exp \left(\alpha_{s,-}(\lambda, t)\right) \overline{\exp \left(\alpha_{s,-}(\lambda, t)\right)}} \frac{W\left(\psi_{-}(\lambda, t), \overline{\psi_{-}(\lambda, t)}\right)}{W\left(\psi_{-}(\lambda, t), \psi_{+}(\lambda, t)\right)}=T(\lambda, 0) .
\end{aligned}
$$

Here we used that the Wronskian $W_{n}(f, g)=a(n)(f(n) g(n+1)-f(n+1) g(n))$ of two solutions satisfying (5.6) does not depend on $n$ or $t$ (see [13, 14, Lemma 12.15]). Similarly,

$$
\begin{aligned}
R_{ \pm}(\lambda, t) & =\frac{\exp \left(\alpha_{s, \pm}(\lambda, t)\right) \exp \left(\alpha_{s, \mp}(\lambda, t)\right)}{\exp \left(\alpha_{s, \mp}(\lambda, t)\right) \exp \left(\overline{\alpha_{s, \pm}(\lambda, t)}\right)} \frac{W\left(\psi_{\mp}(\lambda, t), \overline{\psi_{ \pm}(\lambda, t)}\right)}{W\left(\psi_{ \pm}(\lambda, t), \psi_{\mp}(\lambda, t)\right)} \\
& =\exp \left(\alpha_{s, \pm}(\lambda, t)-\alpha_{s, \mp}(\lambda, t)\right) R_{ \pm}(\lambda, 0) .
\end{aligned}
$$

The time dependence of $\gamma_{ \pm, j}(t)$ follows from $\left\|\psi_{ \pm}\left(\rho_{j}, ., t\right)\right\|=\left\|\hat{U}_{s}(t, 0) \psi_{ \pm}\left(\rho_{j}, ., 0\right)\right\|=$ $\left\|\psi_{ \pm}\left(\rho_{j}, ., 0\right)\right\|$.

A few remarks concerning this theorem are in order.

Remark 5.4. (i) First of all note that the time dependence of $R_{ \pm}(\lambda, t)$ is not continuous at the band edges (except the lowest and highest). In fact, the integrand in (4.12) has a pole whenever $\mu_{j}(0, t)$ hits a band edge. Suppose that $\mu_{j}(0, t)$ gets close to $E_{2 j}$ at $t=t_{1}$. Then (assuming $G_{s}\left(E_{2 j}, 0, t_{1}\right) \neq 0$ for simplicity)

$\mu_{j}(0, t)=E_{2 j}-\left|\nu_{j}\right|^{2}\left(t-t_{1}\right)^{2}+O\left(t-t_{1}\right)^{3}, \quad \nu_{j}=\frac{G_{s}\left(E_{2 j}, 0, t_{1}\right) \prod_{k \neq 2 j} \sqrt{E_{2 j}-E_{k}}}{\prod_{l \neq j}\left(\mu_{j}\left(0, t_{1}\right)-\mu_{l}\left(0, t_{1}\right)\right)}$.

As long as $t<t_{1}$ we have

$$
\lim _{\lambda \downarrow E_{2 j}}\left(\alpha_{s,+}(\lambda, t)-\alpha_{s,-}(\lambda, t)\right)=0 .
$$

But at $t=t_{1}$ we obtain (setting $\lambda=E_{2 j}+\varepsilon^{2}$ and using $\nu_{j}=(-1)^{g-j} \mathbf{i}\left|\nu_{j}\right|$ )

$$
\begin{aligned}
& \lim _{\lambda \downarrow E_{2 j}}\left(\alpha_{s,+}(\lambda, t)-\alpha_{s,-}(\lambda, t)\right)=\lim _{\lambda \downarrow E_{2 j}} 2 R_{2 g+2}^{1 / 2}(\lambda) \int_{0}^{t_{1}} \frac{\hat{G}_{s}(\lambda, 0, x)}{G_{g}(\lambda, 0, x)} d x \\
& =\lim _{\varepsilon \downarrow 0} 2 \int_{0}^{t_{1}} \frac{\nu_{j} \varepsilon}{\varepsilon^{2}+\left|\nu_{j}\right|^{2}\left(x-t_{1}\right)^{2}} d x=2(-1)^{g-j} \lim _{\varepsilon \downarrow 0} \arctan \left(\frac{t_{1}\left|\nu_{j}\right|}{\varepsilon}\right)=(-1)^{g-j} \mathrm{i} \pi .
\end{aligned}
$$

This is in agreement with the conditions

$$
\begin{array}{ll}
\lim _{\lambda \rightarrow E_{l}} R_{2 r+2}^{1 / 2}(\lambda) \frac{R_{ \pm}(\lambda, t)+1}{T(\lambda)}=0, & E_{l} \neq \mu_{j}(0, t), \\
\lim _{\lambda \rightarrow E_{l}} R_{2 r+2}^{1 / 2}(\lambda) \frac{R_{ \pm}(\lambda, t)-1}{T(\lambda)}=0, & E_{l}=\mu_{j}(0, t),
\end{array}
$$

found in 3 . 
(ii) The norming constants $\gamma_{ \pm, j}(t)$ will vanish whenever $\left(\rho_{j}, \pm\right)=\hat{\mu}_{k}(0, t)$. This is important, since the norming constants must cancel the pole of $\tilde{\psi}_{q, \pm}\left(\rho_{j}, n, t\right)$ at these points such that the Gel'fand-Levitan-Marchenko equation remains well defined.

In fact, these poles come from the normalization $\tilde{\psi}_{q, \pm}(z, 0, t)=1$ and this normalization is natural in the constant background case since it renders $\tilde{\psi}_{q, \pm}(z, n, t)$ time-independent. In the (quasi-)periodic background case this is no longer true, and it seems more natural to allow a time-dependent normalization and replace $\tilde{\psi}_{q, \pm}(z, n, t)$ by the time-dependent Baker-Akhiezer functions $\psi_{q, \pm}(z, n, t)$ rendering the norming constants and the reflection coefficient time-independent.

(iii) Finally let us discuss the connection with the different normalizations for $\gamma_{ \pm, j}$ used in [3], respectively [5].

In 3] we have multiplied $\psi_{q, \pm}(\lambda, n, t)$ by a factor to cancel the poles at the Dirichlet eigenvalues. However, since the Dirichlet eigenvalues change sheets during time evolution, this normalization renders $\hat{\psi}_{q, \pm}(\lambda, n, t)$ discontinuous with respect to $t$ and complicates the time evolution of the corresponding norming constants:

$$
\begin{aligned}
\hat{\gamma}_{ \pm, j}(t)= & \hat{\gamma}_{ \pm, j}(0) \exp \left( \pm 2 R_{2 g+2}^{1 / 2}\left(\rho_{j}\right) \int_{0}^{t} \frac{\hat{G}_{s}\left(\rho_{j}, 0, x\right)}{G_{g}\left(\rho_{j}, 0, x\right)} d x\right. \\
& \left. \pm \sum_{l=1}^{g} \int_{0}^{t} \frac{2 \hat{G}_{s}\left(\mu_{l}(x), 0, x\right) R_{2 g+2}^{1 / 2}\left(\mu_{l}(x)\right)}{\left(\rho_{j}-\mu_{l}(x)\right) \prod_{k \neq l}\left(\mu_{l}(x)-\mu_{k}(x)\right)} d x\right) .
\end{aligned}
$$

This clearly justifies our decision to use the freedom of choosing the initial time to avoid the poles in the first place.

If $H_{q}$ is periodic with period $N$, the normalization chosen in 5 is to divide $\psi_{q, \pm}(\lambda, n, t)$ by the square root of $\frac{1}{N} \sum_{n=1}^{N} \psi_{q,+}(z, n) \psi_{q,-}(z, n)$. To get a welldefined real-valued result this object should be positive. However, since one can compute (see [3])

$$
\sum_{n=1}^{N} \psi_{q,+}(z, n) \psi_{q,-}(z, n)=N \prod_{j=1}^{N-1} \frac{z-\lambda_{j}}{z-\mu_{j}}
$$

where $\lambda_{j} \in\left[E_{2 j-1}, E_{2 j}\right]$ are the zeroes of $\omega_{\infty_{-}, \infty_{+}}$, this factor can be negative or even zero. While this choice does give a simple time dependence

$$
\tilde{\gamma}_{ \pm, j}(t)=\tilde{\gamma}_{ \pm, j}(0) \exp \left(\alpha_{s, \pm}\left(\rho_{j}, t\right)-\alpha_{s, \mp}\left(\rho_{j}, t\right)\right),
$$

it does not cancel the poles at the Dirichlet eigenvalues (it just renders poles into square root singularities), plus it introduces additional singularities at $\lambda_{j}$. In particular, it is unsuitable if one of the eigenvalues coincides with some $\lambda_{j}$.

In summary, since Lemma 3.2 ensures that (5.1) remains valid for all $t$ once it holds for the initial condition, we can compute $R_{ \pm}(\lambda, 0)$ and $\gamma_{ \pm, j}(0)$ from $(a(n, 0)$, $b(n, 0))$ and then solve the Gel'fand-Levitan-Marchenko (GLM) equation to obtain the sequences $(a(n, t), b(n, t))$ as in 3 . In this respect the most important ingredient is the time dependence of the kernel of the GLM equation which follows from Theorem 5.3 . 
Theorem 5.5. The time dependence of the kernel of the Gel'fand-LevitanMarchenko equation is given by

$$
\begin{aligned}
F^{ \pm}(m, n, t)= & \frac{1}{\pi} \operatorname{Re} \int_{\lambda \in \sigma\left(H_{q}\right)} R_{ \pm}(\lambda, 0) \psi_{q, \pm}(\lambda, m, t) \psi_{q, \pm}(\lambda, n, t) \frac{G_{g}(\lambda, 0,0)}{R_{2 g+2}^{1 / 2}(\lambda)} d \lambda \\
& +\sum_{j=1}^{q} \gamma_{ \pm, j}(0) \psi_{q, \pm}\left(\rho_{j}, m, t\right) \psi_{q, \pm}\left(\rho_{j}, n, t\right) .
\end{aligned}
$$

Finally note that since the transmission coefficient is time independent we obtain

Corollary 5.6. Let $(a(t), b(t))$ be a solution of the Toda hierarchy such that (5.1) holds. Then the quantities

$$
A=\prod_{j=-\infty}^{\infty} \frac{a(j, t)}{a_{q}(j, t)}
$$

and $\tau_{j}=\operatorname{tr}\left(H^{j}(t)-H_{q}(t)^{j}\right)$, that is,

$$
\begin{aligned}
\tau_{1} & =\sum_{n \in \mathbb{Z}} b(n, t)-b_{q}(n, t) \\
\tau_{2} & =\sum_{n \in \mathbb{Z}} 2\left(a(n, t)^{2}-a_{q}(n, t)^{2}\right)+\left(b(n, t)^{2}-b_{q}(n, t)^{2}\right),
\end{aligned}
$$

etc., are conserved quantities.

Proof. This follows from the fact that the transmission coefficient is the perturbation determinant (in the sense of Krein) of the pair $H_{q}$ and $H$ (see [1]).

\section{ACKNOWLEDGMENTS}

We thank the referee for several valuable suggestions for improving the presentation.

\section{REFERENCES}

[1] A. Boutet de Monvel and I. Egorova, The Toda lattice with step-like initial data. Soliton asymptotics, Inverse Problems 16, No. 4, 955-977 (2000). MR1776477 (2001g:37138)

[2] W. Bulla, F. Gesztesy, H. Holden, and G. Teschl, Algebro-Geometric Quasi-Periodic FiniteGap Solutions of the Toda and Kac-van Moerbeke Hierarchies, Memoirs of the Amer. Math. Soc. 135/641 (1998). MR1432141(99b:58109)

[3] I. Egorova, J. Michor, and G. Teschl, Scattering theory for Jacobi operators with quasiperiodic background, Comm. Math. Phys. 264-3, 811-842 (2006).

[4] L. Faddeev and L. Takhtajan, Hamiltonian Methods in the Theory of Solitons, Springer, Berlin, 1987. MR0905674 (89m:58103)

[5] N. E. Firsova, On the solution of the Cauchy problem for the Korteweg-de Vries equation with initial data being the sum of periodic and rapidly decreasing functions, Mat. Sb., N. Ser. 135(177), No. 2, 261-268 (1988). MR0937811 (90a:35197)

[6] H. Flaschka, On the Toda lattice. II, Progr. Theoret. Phys. 51, 703-716 (1974). MR0408648 (53:12412)

[7] C. S. Gardner, J. M. Green, M. D. Kruskal, and R. M. Miura, A method for solving the Korteweg-de Vries equation, Phys. Rev. Letters 19, 1095-1097 (1967).

[8] E. A. Kuznetsov and A. V. Mikhaulov, Stability of stationary waves in nonlinear weakly dispersive media, Soviet Phys. JETP 40, No. 5, 855-859 (1975). MR0387847 (52:8685)

[9] P. D. Lax, Integrals of nonlinear equations of evolution and solitary waves, Comm. Pure and Appl. Math. 21, 467-490 (1968). MR0235310 (38:3620)

[10] V. A. Marchenko, Sturm-Liouville Operators and Applications, Birkhäuser, Basel, 1986. MR0897106 (88f:34034) 
[11] J. Michor and G. Teschl, Trace formulas for Jacobi operators in connection with scattering theory for quasi-periodic background, Proc. Operator Theory and Applications in Mathematical Physics 2004, J. Janas, et al. (eds.), Oper. Theory Adv. Appl., Birkhäuser, Basel (to appear).

[12] G. Teschl, Inverse scattering transform for the Toda hierarchy, Math. Nach. 202, 163-171 (1999). MR1694723 (2000e:37124)

[13] G. Teschl, On the Toda and Kac-van Moerbeke hierarchies, Math. Z. 231, 325-344 (1999). MR:1703351 (2000f:37105)

[14] G. Teschl, Jacobi Operators and Completely Integrable Nonlinear Lattices, Math. Surv. and Mon. 72, Amer. Math. Soc., Rhode Island, 2000. MR:1711536 (2001b:39019)

[15] M. Toda, Theory of Nonlinear Lattices, 2nd enl. ed., Springer, Berlin, 1989. MR0971987 (89h:58082)

[16] A. Volberg and P. Yuditskii, On the inverse scattering problem for Jacobi Matrices with the Spectrum on an Interval, a finite systems of intervals or a Cantor set of positive length, Commun. Math. Phys. 226, 567-605 (2002). MR1896882 (2003m:47059)

Kharkiv National University, 47 Lenin ave, 61164 Kharkiv, Ukraine

E-mail address: egorova@ilt.kharkov.ua

Faculty of Mathematics, Nordbergstrasse 15, 1090 Wien, Austria - And - International Erwin Schrödinger Institute for Mathematical Physics, Boltzmanngasse 9, 1090 Wien, Austria

E-mail address: Johanna.Michor@esi.ac.at

$U R L:$ http://www.mat.univie.ac.at/〜jmichor/

Faculty of Mathematics, Nordbergstrasse 15, 1090 Wien, Austria - and - International Erwin Schrödinger Institute for Mathematical Physics, Boltzmanngasse 9, 1090 Wien, Austria

E-mail address: Gerald.Teschl@univie.ac.at

URL: http://www.mat.univie.ac.at/〜gerald/ 